\title{
Interactive public digital displays: investigating its use in a high school context
}

\author{
Nuno Otero ${ }^{\S^{*}}$, Rui José ${ }^{\S}$, and Bruno Silva ${ }^{\S}$ \\ ${ }^{\S}$ Centro Algoritmi, University of Minho, Campus de Azurém, Guimarães, Portugal \\ * Department of Media Technology, Linnæus University, Sweden \\ \{nuno.otero; rui; bruno.silva\}@dsi.uminho.pt
}

\begin{abstract}
This paper presents a longitudinal user study that investigated the adoption of some Bluetooth based functionalities for a public digital display in a high school. More specifically, the utilization of Bluetooth device naming extended beyond social identity representation and introduced the use of a simple interaction mechanism. The interaction mechanism involves recognizing parts of the Bluetooth device name as explicit instructions to trigger the generation of content on an interactive public display. Together with representatives of the teachers' community, the design team defined some social rules concerning usage in order to account for the specificities of the place. In the user study, three fully functional prototypes were deployed at the school hall of the high school. The functionalities introduced with the different prototypes were: the visualization on the display of the Bluetooth device names, the possibility to contribute to tag clouds and the possibility to choose icons from a given set for self-expression. The results suggest that people appropriated some but not all of the functionalities employed. Implications of our findings to the design of interactive digital displays are pointed out.
\end{abstract}

Keywords. Ubiquitous computing; digital public displays; bluetooth, user studies

\section{Introduction}

Our on-going long-term research goal concerns the investigation of the digital displays' design space to support people's interactions through these artefacts in public spaces. People are taking advantage of new web, mobile and ubiquitous technologies to explore novel ways to interact in complex social situations $[4,9,16]$. Digital displays can be an important technology for many types of ubiquitous computing scenarios since it can provide a simple and effective way to bring digital information into our public spaces. Furthermore, providing interactivity in digital displays can be used to foster user-generated pervasive content back to the virtual world. However, research has highlighted that enticing people to participate and explore the potential of the systems is a major challenge [1,5]. Furthermore, there are complex issues related with publication management [6]. For example, the design of situated displays is fundamentally affected by a trade-off on control sharing. On the one hand, the need to 
support a wide range of practices and social settings around the display suggests approaches that build strongly on active user participation and high levels of appropriation. On the other hand, the expected convergence towards the social practices of the community as a whole, suggests approaches such as mediation and explicit user permissions that define more rigidly the purpose of the system.

The prototypes presented in this paper involve the scanning and depiction of Bluetooth device names in digital displays situated in public spaces. More specifically, using simple techniques involving the parsing of the device names, we were able to provide functionalities that not only served to communicate some sort of social identity but also to trigger particular types of interaction: influencing tag clouds and expressing preferences towards content.

This present work investigates how people sharing a particular place within a high school appropriated a set of Bluetooh based interactive mechanisms. Given the nature of the study we opted to consider research questions instead of fully-fledged hypotheses. The research questions are:

- Did people change the name of their device and created an individual public presence on the display?

- Did people use the tag clouds and icons functionalities (corresponding to the functionalities introduced with prototype 2 and 3 - see below the descriptions)?

- Did some of the features of the prototypes related to the display of content influence organizational practices?

The remainder of the paper goes as follows. Section two provides an overview of related work, focusing on situated public displays. Section three describes the framing of the study, including the prototype deployed and its functionalities. Section four presents the results and section five the overall discussion. In section six we present the lessons learned and future developments.

\section{Background}

The display of Bluetooth presence in public or semi-public displays has been explored in a variety of systems. Some studies have investigated Bluetooth scanning as a mechanism for sensing presence and uncovering all sorts of social patterns, e.g. the familiarity of the surrounding environment [15], the social situation [13], and more general large-scale reality mining [3]. The Cityware project [10] looked at several ways of leveraging the capture of information regarding Bluetooth mobility, including a set of in-situ visualizations about current or recent Bluetooth presences. The system supports links between Bluetooth devices and the Facebook identities of their owners, as a way to create a link between physical presence and virtual presence. The system uses in-situ presence information as a way to generate content for the virtual world. More specifically, it provides data to a Facebook application that lets people associate physical co-presence information with their social network.

Another example of the use of presence as a driver for situated interaction around public displays is the Proactive displays system $[11,12]$. The detection of nearby 
RFID tags was used as a trigger for showing profile information about the owner of the tag, in an attempt to promote occasional encounters between people around the display. However, this approach requires a priori definition of individual profiles with associated data and assumes that everyone will be using a particular type of tag. Furthermore, people have a very limited role in the system, which is basically to move around and be detected. The Bluescreen project, in its turn, explores the use of Bluetooth presence to optimise the selection of adverts for display [8]. Content that has already been shown when a particular Bluetooth device was present is avoided if that device is present again, thus reducing the likelihood of the same content being shown again to the same person.

In relation to research that specifically address people's usage of Bluetooth functionality and their appropriation to extend their social everyday practices the following two examples are particularly relevant. O'Neill et al. [9] investigated the use of Bluetooth and the naming of devices through the scanning of device names in public spaces. In their study they were able to classify distinct types of device names and proposed that people's usage of Bluetooth can be seen as an example of the emergence of a specific culture around artifact utilization. Kindberg and Jones [9] went beyond the simple scanning of device names and, through 29 semi-structured interviews, tried to uncover the meaning behind the naming practices. Kindberg and Jones [9] study revealed that people tend to use Bluetooth mainly to share files. Sometimes, however, people also choose device names that reflected their presences in other social circles: adopting the same name as the online one or choosing the same name that identifies them in particular practices. Kindberg and Jones [9] also report that most of their interviewed participants did not tend to change their device name frequently. The use of Bluetooth names for conveying simple commands to public displays has been studied by Jose et al. as part of the instant places system [7] and also by Davies et al [2] as part of the Lancaster e-campus system. Even though the interaction approach is essentially the same as in our prototype, these studies were both focused on the ability to support spontaneous interaction with the displays, and did not addressed the issues of control sharing involved.

\section{$3 \quad$ Methodology}

The methodology followed for the study involved the deployment of fully functional prototypes in a real world context and the provision of information about the functionalities of the system without suggesting particular ways of usage. The study ran for approximately 24 weeks. The next sub-sections will describe the different prototypes deployed, the setting of the study and the methods utilized.

\subsection{The prototypes}

The prototypes deployed at the school included a public display in which content was generated, directly or indirectly, from Bluetooth presence. Generally speaking, the system comprises a Bluetooth enabled computer connected to a public screen and 
linked to a central repository. Information about nearby devices is periodically collected by a Bluetooth scanner and fed to a situation data model that manages data about the place and present devices. The central repository maintains persistent information about previous sessions, and combines information from pervasively distributed data sources, allowing for multiple screens in a large space to share the same presence view. The system does not need any a priori information about people, their profiles, permissions or groups, as all the information in the repository is entirely created from the history of presences. All the prototypes also included some specific content suggested by the school team, the most relevant being the school news feeds.

The basic form of interaction with the first prototype of the system is to have a discoverable Bluetooth device with its name shown on the public display. This can be viewed as an implicit form of interaction where a person unexpectedly finds his or her name on the display. However, it can quickly turn into an explicit form of interaction when that person changes the device name for visualization on the screen. The visualization of the Bluetooth presences provides an element of situation awareness that we hoped would foster the use of Bluetooth naming as a way for self-expression. In order to explore further this latter functionality, the system supported the use of simple commands in the Bluetooth device names by parsing the device names in search for keywords that were recognised as commands ( 't.word' for tag clouds and 'g.word' for icons) and then using them to trigger specific actions. For the second prototype the added functionality was the introduction of tag clouds. In the third prototype the users were also able to choose icons from a specific set and displayed it next to his/her device name as a way to express some sort of individual preference.

\subsection{The place}

The user study took place at a high school. As most high schools, this place is a vibrant space full of activities, where students, teachers and other supporting personnel meet daily. The school is divided into several distinct pavilions with different purposes (classrooms, refectory, administration, students' common room etc). After initial consultations with the school representatives it was deemed appropriate to install the digital display at the entrance hall of the administrative pavilion. The administrative pavilion contains the school office, the teachers' common room, the school library and other administrative offices. Very different groups of people use this pavilion in distinct ways. Teachers utilize their common room between teaching periods, usually staying for short bursts of around ten minutes and longer during lunch break. Administrative personnel, however, tend to spend their working day within their office. Students and their parents can go to the teachers' common room and to the school office for specific meetings and dealings, varying a lot the amount of time they spent at this location. The library activities frequently involve longer stays. Regarding the particular location where the display was installed, the entrance, it seems reasonable to consider that people do not tend to stay there for long periods but is frequently accessed. This means that Bluetooth scanning will probably pick this constellation of parallel occurrences but the actual time people tend to spend in the visualization of the display is bound to some passers-by place specificities just described. 


\subsection{The methods followed in the design/development cycle}

\section{Meetings with teachers}

Several meetings took place between the design team and representatives of the school. In the beginning of the intervention, a meeting with the school's Director was set and aimed at: (a) presenting the general ideas behind the project, including the envisioned system's functionalities, and defining the general scope of the users' studies; and (b) agreeing on a particular line of action that involved the creation of a teachers' team to follow the project, provide the necessary support and vouch the different activities to be pursued. After this initial step with the school's Director, a school teachers' team was appointed by the school's Director to follow the project. More specifically the teachers' team was in charge of:

- Discussing with the design team the specifics of the prototypes' functionalities, approving the deployment of the different prototypes and controlling the correct usage of the system.

- Ensuring that the different school's content channels to feed the display were updated with relevant information.

- Checking the appropriateness of the Bluetooth device names in order to approve the different identities that were being created within the system. More specifically, the teachers' team was responsible for checking lists of scanned device names and approve them for publication on the digital display.

\section{Collecting system logs}

We collected system logs over 24 weeks. The logs collected MAC addresses of the different devices within the range of the Bluetooth scanner, as well as the corresponding device names. This means that the database created can keep track of the different sightings of devices and the possible change of device names that, in turn, can correspond to the use of some of the functionalities provided (or just a change of the way the owner of the device decides to present himself to the system).

The data collected can be divided into the following distinct periods:

- Silent scanning - to begin with we installed a Bluetooth scanner just to collect the usual activity regarding Bluetooth usage in order to understand better what would be the changes of introducing our system. This period corresponded to the first five weeks of the study.

- First prototype - On week 7 we deployed the first prototype (see description above).

- $\quad$ Second prototype - On week 17 the second prototype was made available.

- Third prototype - Finally, on week 21 the third prototype with the remaining of the functionalities developed was introduced. 


\section{$4 \quad$ Results}

Table 1 shows the number of device names submitted for approval and its actual approval by the school's team. At the end of the silent scanning period the first list of device names collected was submitted for approval to the school's team (see subsection "Meeting with teachers"), so that the device names that would be displayed initially with the first prototype conformed to school's norms. From Table 1 we can see that most of the unapproved device names are found in lists two and three. This is expected since it is the period where people are trying out the system and see if they can "win" and display inappropriate terms. What such behaviour also suggests is that people are trying to take advantage of the relative anonymity provided by the system in order to stretch social rules. Nevertheless, the number of inappropriate device names drops sharply: in the 4th list only 3 device names are not approved.

Table 1 - Number of device names sent and approved per period of the study

\begin{tabular}{|c|c|c|}
\hline Period & Sent & Approved \\
\hline Silent scanning & $\begin{array}{l}\text { 1st list: week } 6 \text { (232 device } \\
\text { names submitted) }\end{array}$ & $\begin{array}{l}\text { 1st list: week } 6 \text { (231 device names } \\
\text { approved - 99\%) }\end{array}$ \\
\hline First prototype & $\begin{array}{l}\text { 2nd list: week } 9 \text { (202 device } \\
\text { names submitted) } \\
\text { 3rd list: week } 13 \text { (130 device } \\
\text { names submitted) }\end{array}$ & $\begin{array}{l}\text { 2nd list: week } 9 \text { (181 device names } \\
\text { approved - 90\%) } \\
\text { 3rd list: week } 13 \text { (107 device names } \\
\text { approved - 82\%) }\end{array}$ \\
\hline Second prototype & $\begin{array}{l}\text { 4th list: week } 18 \text { (102 device } \\
\text { names submitted) }\end{array}$ & $\begin{array}{l}\text { 4th list: week } 18 \text { (99 device names } \\
\text { approved - 97\%) }\end{array}$ \\
\hline Third prototype & $\begin{array}{l}\text { 5th list: week } 23 \text { (155 device } \\
\text { names submitted) }\end{array}$ & 5th list: no response \\
\hline
\end{tabular}

The data collected using the system logs and displayed in Table 2 allow the inspection of how many devices were detected and how many times people changed their device name in the different periods under investigation. In relation to the number of devices detected, Table 2 shows an increase from the silent scanning period to the period corresponding to the two first weeks of the first prototype's deployment. However, after this initial period of adoption one can see fluctuations of usage (note, however, that in some weeks the numbers of devices spotted drops probably due to school holidays). Regarding the device names the first two weeks after the deployment of the first prototype is also the particular time window that seems to show people "experimenting" with their Bluetooth presence on the display. Most of the device names chosen (and approved) correspond to first names or nicknames. None of the users tried to change their icon.

Table 3 shows some indicators regarding the use of the tag cloud functionality. In total it seems that 39 people tried to take advantage of the tag cloud. This number corresponds approximately to one fourth of the people using the system in the corresponding period (the second prototype). However, we did register some problems with the syntax for a correct utilization since there were 18 mistakes detected coming from 13 distinct devices. 
Table 2 - Number of unique devices and names collected during the silent scanning and deployments periods

\begin{tabular}{|c|c|c|c|c|}
\hline Prototype & Week & Unique devices & Unique device names & Nr devices $>1$ name \\
\hline \multirow[t]{7}{*}{ Silent Scanning } & 2 & 74 & 74 & 0 \\
\hline & 3 & 95 & 96 & 1 \\
\hline & 4 & 78 & 78 & 0 \\
\hline & 5 & 98 & 98 & 0 \\
\hline & 6 & 97 & 97 & 0 \\
\hline & 7 & 90 & 93 & 3 \\
\hline & Total & 259 & 267 & 8 \\
\hline \multirow[t]{12}{*}{ First prototype } & $7+8$ & 148 & 181 & 23 \\
\hline & 9 & 169 & 196 & 16 \\
\hline & 10 & 99 & 113 & 5 \\
\hline & 11 & 110 & 125 & 7 \\
\hline & 12 & 112 & 119 & 6 \\
\hline & 13 & 102 & 104 & 2 \\
\hline & 14 & 40 & 42 & 2 \\
\hline & 15 & 21 & 21 & 0 \\
\hline & 16 & 93 & 94 & 1 \\
\hline & 17 & 97 & 98 & 1 \\
\hline & 18 & 126 & 127 & 1 \\
\hline & Total & 504 & 628 & 74 \\
\hline \multirow{3}{*}{$\begin{array}{c}\text { Second proto- } \\
\text { type }\end{array}$} & 19 & 112 & 114 & 2 \\
\hline & $20+21$ & 106 & 109 & 3 \\
\hline & Total & 164 & 169 & 5 \\
\hline \multirow[t]{5}{*}{ Third prototype } & 22 & 161 & 165 & 4 \\
\hline & $23+24$ & 131 & 135 & 4 \\
\hline & Total & 219 & 229 & 9 \\
\hline & $\begin{array}{c}\text { All Prototypes } \\
\text { Total }\end{array}$ & 655 & 818 & 101 \\
\hline & Total & 763 & 955 & 121 \\
\hline
\end{tabular}

Notes - (1) In column "Nr devices $>1$ " the difference between the sum of week's partial results and the period's total can be explained by considering that some users probably changed their device name between weeks and outside the rage detection of the Bluetooth scanner. Also to note that not always the difference between the total of unique devices and the total of unique device names is equal to the number of devices with more than one name. This is easily explained by users that changed their device name more than once. (2) Weeks $7+8,20+21$ and $23+24$ show aggregated results due to some technical problems that occured.

The news feeds provided by the school to be shown in the public display can give us a glimpse of the acceptance of the new artefact among the school's more formal organizational units/structure. Several school departments initially agreed to contribute. However, in many cases their actual involvement was not consistent over the 
time. In fact, most of the updates of the content happened during the first two months after the deployment of the first prototype. This suggests some kind of novelty effect that faded as time went by.

Table 3 - Number of tags used during the study. Valid and invalid tags correspond to tags with a well formed or incorrect system syntax

\begin{tabular}{|c|c|c|}
\hline Tags & Number of used tags & Devices \\
\hline Valid tags & 29 & 26 \\
\hline Invalid tags & 18 & 13 \\
\hline
\end{tabular}

\section{Discussion}

The results reported suggest that people sharing the place did adopt some of the functionalities provided but not all. This seems to be in line with the views by Brignull et al. [1] and Huang et al [5] that enticing people to interact with public displays remains a challenge. The remaining of the discussion follows the research questions stated above.

Did people change the name of their device and created an individual public presence on the display?

The results regarding the change of device names strongly suggest that people did create a presence to be displayed since the comparison of the visible device names between the silent scanning period and the periods corresponding to the deployment of the first prototype clearly shows an increase. In a previous study José and Otero [7] reported a different pattern of usage of the device name functionality: users were able to utilize this functionality in order to use the display as a message board. However, in this present study we did not observe the same phenomena and we believe this is due to the approvals' procedure set. The lag between changing the device name and the actual approval, with its consequent appearance on the display, makes the exchange of messages on the spot impossible. Furthermore, we should also note that the possible messages would only be displayed if the devices were detected and so leaving messages for others to see asynchronously was not possible as well unless the device was detected once again. In fact, considering the policy implemented by the school team to approve device names it seems reasonable to assume that any messaging system would have to undergo a similar approval procedure.

These considerations seem to highlight the point made regarding the need to consider carefully the issue of control sharing. In this present case the control was clearly centralized but this does not seem to be the best option if one intends to create a highly dynamic display, content wise. More research is needed regarding the mechanisms that can be put into place to allow a satisfactory control of the content without jeopardizing people's creative appropriations of the display.

Did people use the tag clouds and icons functionalities?

Our results show that tag clouds were used but people did not adopt the icons as a way to express themselves. The tag cloud functionality was set as a way to people 
express their preferences regarding some types of affiliation (like football clubs and school classes). In some way this functionality is working at a group identity level and the symbols are clearly defined and shared. However, people probably assumed that the icons are related to a more individual level of identification and they might prefer to create their own icons instead of having to choose from a pre-defined set. If this is indeed the case then there is a need to construct a distinct procedure that allows a less restrictive self-expression. Once again, however, we are touching the problem of control sharing.

Did some of the features of the prototypes related to the display of content influence organizational practices?

In relation to the organizational practices, two issues seem to be particularly relevant: the procedure set for device names' approval and the actual provision of news feeds to be displayed. The procedure put in place to approve the device names is fairly centralized in the school's team. This, of course, puts some pressure on the team's members since it is an extra task they have to engage with on top of their usual school activities. In turn this provoked some delays on the approvals that might have a negative influence on people's experimentation with distinct displayed identities. Once again, the same content regarding control sharing is relevant.

In relation to the news feeds, the initial response was very promising but the fading of the updates once again reveal that extra work needs a perceived added value. In other words, it seems to us that the centralized model of control sharing assumed in the school influenced the way the display was thought to be useful and created mechanisms that were not particularly successful in terms of a more spontaneous adoption by the community sharing the place.

\section{Conclusions}

The results show that people can be enticed to interact through digital public displays and, maybe, contribute to the construction of an extended understanding of the place by reaching into the digital world. However, our study also suggests that not all interaction mechanisms and procedures to generate content are suitable to all types of places. In other words, it seems that one size fits all type of solution is clearly not effective or efficient. More research is needed in order to understand the specificities of a particular place and how to match these with the appropriate interaction mechanisms.

\section{Acknowledgements}

The research leading to these results has received funding from FCT under the Carnegie Mellon - Portugal agreement: WESP (Web Securityand Privacy (Grant CMU-PT/SE/028/2008). 


\section{$7 \quad$ References}

1. Brignull, H. et al. 2004. The introduction of a shared interactive surface into a communal space. Proceedings of the 2004 ACM conference on Computer supported cooperative work. (2004), 49-58.

2. Davies, N. et al. 2009. Using bluetooth device names to support interaction in smart environments. Proceedings of the 7th international conference on Mobile systems, applications, and services (New York, NY, USA, 2009), 151-164.

3. Eagle, N. and Pentland, A. 2006. Reality Mining: Sensing Complex Social Systems. Personal and Ubiquitous Computing. 10, 4 (2006).

4. Hardey, M. 2002. Life Beyond the Screen: Embodiment and Identity Through the Internet. The Sociological Review. 50, 4 (Nov. 2002), 570-585.

5. Huang, E.M. et al. 2006. Secrets to Success and Fatal Flaws: The Design of Large-Display Groupware. IEEE Computer Graphics and Applications. 26, 1 (2006), 37-45.

6. José, R. et al. 2012. Beyond interaction: Tools and practices for situated publication in display networks. (Porto, Portugal, 2012).

7. José, R. et al. 2008. Instant Places: Using Bluetooth for Situated Interaction in Public Displays. IEEE Pervasive Computing. 7, 4 (2008), 52-57.

8. Karam, M. et al. 2007. Evaluating BluScreen: Usability for Intelligent Pervasive Displays. The Second IEEE International Conference on Pervasive Computing and Applications (ICPCA '07) (Birmingham, UK., 2007).

9. Kindberg, T. and Jones, T. 2007. 'Merolyn the Phone': A Study of Bluetooth Naming Practices. UbiComp 2007: Ubiquitous Computing. 318-335.

10. Kostakos, V. and O'Neill, E. 2008. Cityware: Urban computing to bridge online and real-world social networks. Handbook of Research on Urban Informatics: The Practice and Promise of the Real-Time City. (2008), 195-204.

11. McCarthy, J.F. et al. 2003. Proactive Displays \& The Experience UbiComp Project. First International workshop on Ubiquitous Systems for Supporting Social Interaction and Face-to-Face Communication in Public Places (Seattle, Washington, USA, 2003).

12. McDonald, D.W. et al. 2008. Proactive displays: Supporting awareness in fluid social environments. ACM Trans. Comput.-Hum. Interact. 14, 4 (2008), 1-31.

13. Nicolai, T. et al. 2006. Exploring Social Context with the Wireless Rope. 1st International Workshop on MObile and NEtworking Technologies for social applications (MONET'06) (Montpellier, France, 2006).

14. O'Neill, E. et al. 2006. Instrumenting the city: Developing methods for observing and understanding the digital cityscape. UbiComp 2006: Ubiquitous Computing. (2006), 315-332.

15. Paulos, E. and Goodman, E. 2004. The familiar stranger: anxiety, comfort, and play in public places. Proceedings of the SIGCHI conference on Human factors in computing systems. (2004), 223-230.

16. Turkle, S. 1995. Life on the Screen: Identity in the Age of the Internet. Simon and Schuster Trade. 